\title{
The Fine Structure Constant and Radiative Corrections for the Electron and Positron Cores as Viewed in the Planck Vacuum Theory
}

\author{
William C. Daywitt
}

\begin{abstract}
The present paper derives the radiative corrections for the electron and positron cores in the Planck vacuum theory, leading to the electron and position particles in a 7-dimension spacetime. The physical meaning of the fine structure constant is twofold: it is the ratio of the electron spin coefficient to the electron-core spin coefficient; and it is the probability that an electron or positron will emit or absorb a photon. The nature of this photon defines the spin quanta in the PV theory. Results suggest that the Feynman and Schwinger QED calculations refer to the PV state. Finally, the closed-loop electron-positron pairs in the Feynman diagrams are explained.
\end{abstract}

Index Terms-Fine Structure Constant, Radiative Corrections, Dirac Equation, Electron, Positron, Particle-Antiparticle Pair, Spin Quantum, Planck Vacuum, Vacuum Structure.

\section{INTRODUCTION}

The theoretical foundation [1] [2] [3] of the PV theory rests upon the unification of the Einstein, Newton, and Coulomb superforces:

$$
\frac{c^{4}}{G}\left(=\frac{m_{*} c^{2}}{r_{*}}\right)=\frac{m_{*}^{2} G}{r_{*}^{2}}=\frac{e_{*}^{2}}{r_{*}^{2}}
$$

where the ratio $c^{4} / G$ is the curvature superforce that appears in the Einstein field equations. $G$ is Newton's gravitational constant, $c$ is the speed of light, $m_{*}$ and $r_{*}$ are the Planck mass and length respectively [4, p.1234], and $e_{*}$ is the bare (or coupling) charge. The fine structure constant is $\alpha \equiv e^{2} / e_{*}^{2}$, where $e$ is the observed electronic charge magnitude.

The particle/PV coupling force

$$
F_{e}(r)=\frac{e_{*}^{2}}{r^{2}}-\frac{m_{e} c^{2}}{r}
$$

the electron core $\left(-e_{*}, m_{e}\right)$ exerts on the invisible PV state; along with its coupling constant

$$
F_{e}\left(r_{e}\right)=0
$$

and resulting Compton radius

$$
r_{e}=\frac{e_{*}^{2}}{m_{e} c^{2}}
$$

lead to the important string of Compton relations

$$
r_{e} m_{e} c^{2}=e_{*}^{2}=r_{*} m_{*} c^{2} \quad(=c \hbar)
$$

DOI: http://dx.doi.org/10.24018/ejers.2020.5.5.1904

Published on May 3, 2020.

William C. Daywitt is retired from the National Institute of Standards and Technology, Boulder, CO 80305 USA (e-mail: wcdaywitt@me.com). for the electron and positron cores, where $\hbar$ is the reduced Planck constant. The Planck particle Compton radius is $r_{*}=$ $e_{*}^{2} / m_{*} c^{2}$, which is derived by equating the Einstein and Coulomb superforces from (1). To reiterate, the equation in (2) represents the force the free electron core exerts on the invisible PV space, a space that is itself pervaded by a degenerate collection of Planck-particle cores $\left( \pm e_{*}, m_{*}\right)$ [5]. The positron core is $\left(+e_{*}, m_{e}\right)$. It should be noted that all of the constants in (5) are Lorentz invariant. The Lorentz invariance of the coupling constant in (3) leads to the energy and momentum operators of the quantum theory [5].

Section II expresses the covariant Dirac equation in terms of PV parameters. Section III discusses radiative corrections. Section IV presents some comments and conclusions and Appendix B discusses the intrinsic magnetic moment.

\section{CORE EQuATIONS}

The Dirac particles defined in the present paper are the electron core and its antiparticle, where the following Dirac equations are assumed to result from the equation in (2) operating on the PV state. This assumption follows from the appearance of $e_{*}^{2}$ and $m_{e}$ in the following Dirac equations, where $m_{e}$ is the electron rest mass.

Using (5), the covariant form [6, p.90] [Appendix A] of the Dirac equation for the electron and positron cores combined can be expressed as:

$$
\begin{aligned}
& \left(i \hbar \gamma^{\mu} \frac{\partial}{\partial x^{\mu}}-m_{e} c\right) \psi=\left(i \frac{e_{*}^{2}}{c} \gamma^{\mu} \frac{\partial}{\partial x^{\mu}}-m_{e} c\right) \psi= \\
& {\left[i \frac{e_{*}^{2}}{c} \gamma^{0} \frac{\partial}{\partial x^{0}}+i \gamma^{0}\left(\begin{array}{cc}
0 & S_{j} \\
S_{j} & 0
\end{array}\right) \frac{\partial}{\partial x^{j}}-m_{e} c\right] \psi=0}
\end{aligned}
$$

where the second term in (6) is summed over $j=1,2,3$. The relativistic spin $\vec{S}$ is

$$
\vec{S}=\frac{e_{*}^{2}}{c} \vec{\sigma} \quad \text { with } \quad \vec{\mu}=e r_{e} \vec{S}
$$

where the second equation is the intrinsic magnetic moment (Appendix B) for the electron (9) and positron (10) core equations and the Dirac equation (6).

Using

$$
\psi=\left(\begin{array}{l}
u \\
v
\end{array}\right)
$$

in a previous paper [7], the above Dirac equation is reduced to two particle-antiparticle equations, one

$$
i \frac{\left[\left(-e_{*}\right)\left(-e_{*}\right)\right]}{c}\left(\frac{\partial u}{\partial x^{0}}+\sigma_{j} \frac{\partial v}{\partial x^{j}}\right)=m_{e} c u
$$


for the electron core, and one

$$
i \frac{\left[\left(e_{*}\right)\left(-e_{*}\right)\right]}{c}\left(\frac{\partial v}{\partial x^{0}}+\sigma_{j} \frac{\partial u}{\partial x^{j}}\right)=m_{e} c v
$$

for the positron core, where $u$ and $v$ are the $2 \times 1$ spinor wavefunction solutions to the equations. This notation $\left[\left(e_{1}\right)\left(e_{2}\right)\right]$ indicates that $\left(e_{1}\right)$ represents the free-space electron or positron charge, and $\left(e_{2}\right)$ represents the charges in the negative PV branch $\left(-e_{*}, m_{*}\right)$. The two particles belonging to (9) and (10) belong in two separate 4-dimensional spacetimes [7]. If they are forced into the same spacetime, they will annihilate one another.

\section{RADIATIVE CORRECTIONS}

This section opens with the following quote [8, p.129] from Feynman: "There is a most profound and beautiful question associated with the coupling constant, $e$ - the amplitude for a real electron to emit or absorb a real photon. It is a simple number that has been experimentally determined to be close to -0.08542455 . (My physicist friends won't recognize this number, because they like to remember it as the inverse of its square: about 137.03597 with an uncertainty of about 2 in the last decimal place. It [the fine structure constant] has been a mystery ever since it was first discovered more than fifty years ago, and all good theoretical physicists put this number up on their wall and worry about it.)" The salient comment here is that " $e$ - [is] the amplitude for a real electron to emit or absorb a real photon". The term "real electron" is understood in the present paper to mean equation (9) with its radiative corrections, where the second equation in (13) shows the first order correction in $\alpha$.

Central to the radiative spin corrections is the fine structure constant

$$
\alpha \equiv \frac{e^{2}}{e_{*}^{2}}=\frac{e^{2} / c}{e_{*}^{2} / c} \approx \frac{1}{137}
$$

where $e^{2} / c$ and $e_{*}^{2} / c$ are respectively the electron and electroncore spin coefficients. Then, from the final comment in the preceding paragraph, $\alpha$ must be the probability (amplitude squared) that an electron will emit or absorb a photon. This probability is essential for understanding the radiative corrections to follow.

When radiative corrections are applied to $\vec{\mu}: e^{2}$-photons and PV particles scatter off each other within vacuum state, leading to a small increase in the electron-core g-factor. Using $\alpha^{-1}=$ 137.0 [9, p.722] for the inverse fine structure constant in the Schwinger QED approximation [10] [6, p.298], the relative change in the magnetic moment (Appendix B) is

$$
\begin{gathered}
\frac{\delta \mu}{\mu}=\frac{g}{2}-1=\frac{e^{2}}{2 \pi c \hbar}=\frac{1}{2 \pi} \frac{e^{2} / c}{e_{*}^{2} / c} \\
=\frac{\alpha}{2 \pi}=0.001162
\end{gathered}
$$

leading to

$$
g=2\left(1+\frac{\alpha}{2 \pi}\right) \quad \text { and } \quad \vec{\mu}=\left(1+\frac{\alpha}{2 \pi}\right) e r_{e} \vec{S} .
$$

Equations (11) and (13) suggest that the Feynman [8, p.129] and Schwinger [10] calculations refer the PV state.

\section{COMMENTS AND CONCLUSIONS}

Equations (11) and (13) are the principal results of the present paper: the ratio $\alpha$ is an emission-absorption probability; and, as such, the radiative corrections (13) for the electron and positron cores center around that ratio. If $\alpha$ were to vanish, there would no corrections. In other words, if the magnetic moment $\vec{\mu}$ does not interact (exchange photons) with the PV state, there can be no radiative corrections. The corresponding spin quanta for the photons are included in the products

$$
\alpha \hbar \omega=\alpha \frac{e_{*}^{2}}{c} \omega=\frac{e^{2}}{c} \omega .
$$

Using (5), the approximation in (12) can be expresses as

$$
\frac{\alpha}{2 \pi}=\frac{e^{2} / 2 \pi r_{e}}{m_{e} c^{2}}=\frac{e^{2} / 2 \pi r_{*}}{m_{*} c^{2}}
$$

where all the parameters in (15) except $e^{2}$ are recognizable PV parameters: $2 \pi r_{e}\left(2 \pi r_{*}\right)$ is the "circumference" of the sphere of "radius" $r_{e}\left(r_{*}\right)$ that contains the particle mass energy $m_{e} c^{2}$ $\left(m_{*} c^{2}\right)$. It is interesting to note where the second and third ratios in (15) are applicable: the second applies anywhere within the quasi-continuum of the PV theory; while the third refers to the discontinuous degenerate collection of Planckparticle cores that constitute the Planck-particle pervasion of that continuum. A corollary to that note is: if the radiative corrections are to be expressed as a power series in $\alpha$, it should be carried out at the Planck level where the third ratio applies.

It is noted in closing that: the closed-loop photon disintegrations into an electron-position pair in Figures 75 and 76 of [8, pp.117-118] correspond, in the PV theory [7], to the momentary excitations of the positron (in a separate 4-dimension spacetime) into the well-known 4-dimensional spacetime of the corresponding electron excitation, with the subsequent pair annihilation creating the final photon that terminates the electron-positron loop. Energy and momentum conservation [6, p.275] are assured by the presence of Planck particle cores in the vicinity of the excitations. It is concluded then, that the spin quanta are created and annihilated within the vacuum state.

\section{APPENDIX A}

THE $\gamma$ AND $\beta$ MATRICES

The $4 \times 4 \gamma, \beta$, and $\alpha_{j}$ matrices used in the Dirac theory are defined here: where $[6, \mathrm{p} .91]$

$$
\gamma^{0} \equiv \beta=\left(\begin{array}{cc}
I & 0 \\
0 & -I
\end{array}\right)
$$

and $(j=1,2,3)$

$$
\gamma^{j} \equiv \beta \alpha_{j}=\left(\begin{array}{cc}
0 & \sigma_{j} \\
-\sigma_{j} & 0
\end{array}\right)
$$

and where $I$ is the $2 \times 2$ unit matrix and

$$
\alpha_{j}=\left(\begin{array}{cc}
0 & \sigma_{j} \\
\sigma_{j} & 0
\end{array}\right)
$$

where the $\sigma_{j}$ are the $2 \times 2$ Pauli spin matrices

$$
\sigma_{1}=\left(\begin{array}{cc}
0 & 1 \\
1 & 0
\end{array}\right), \sigma_{2}=\left(\begin{array}{cc}
0 & -i \\
i & 0
\end{array}\right), \sigma_{3}=\left(\begin{array}{cc}
1 & 0 \\
0 & -1
\end{array}\right)
$$


and $\boldsymbol{\alpha}=\left(\alpha_{1}, \alpha_{2}, \alpha_{3}\right)$. The zeros in (A1)-(A3) and (A5) are $2 \times 2$ null matrices.

The $m_{e} c$ in (6) represents the $4 \times 4$ matrix

$$
m_{e} c\left(\begin{array}{cc}
I & 0 \\
0 & I
\end{array}\right) .
$$

The zero on the right side of (6) represents the $4 \times 4$ null matrix and the zeros in the $\vec{S}$ matrix represent $2 \times 2$ null matrices. The $S_{j}$ and $\sigma_{j}$ in (6) are $2 \times 2$ matrices; so their parenthesis represents a $4 \times 4$ matrix.

The coordinates $x^{\mu}$ are

$$
x^{\mu}=\left(x^{0}, x^{1}, x^{2}, x^{3}\right)
$$

where $x^{0} \equiv c t$.

\section{APPENDIX B}

\section{INTRINSIC MAGNETIC MOMENT}

The difference between the core equation and the particle equation (if it exists) is its intrinsic magnetic moment. The particle equation includes radiative corrections, while the core equation does not.

In general (radiative corrections or not), the intrinsic magnetic moment $\vec{\mu}$ is related to the spin vector $\vec{S}$ through the equations $[6, \mathrm{p} .81]$

$$
\vec{\mu}=g \mu_{B} \vec{S}=g \frac{e r_{e}}{2} \vec{S}
$$

where $g$ is the g-factor and $\mu_{B}$ is the Bohr magneton

$$
\mu_{B}=\frac{e \hbar}{2 m_{e} c}=\frac{e c \hbar}{2 m_{e} c^{2}}=\frac{e e_{*}^{2}}{2 m_{e} c^{2}}=\frac{e r_{e}}{2}
$$

and where $e r_{e}$ is the magnetic dipole moment. Although the g-factor is exactly 2 for the core equations, there is an anomalous-moment increase to this value due to radiative corrections. For $g=2$, (B1) yields

$$
\vec{\mu}=\operatorname{er}_{e} \vec{S}
$$

for the intrinsic magnetic moment for the electron and positron cores and the Dirac equation. The sign of $e$ is, of course, that of $\left(e_{1}\right)$ in $(9)$ or $(10)$.

\section{REFERENCES}

[1] P. Davies, Superforce: the Search for a Grand Unified Theory of Nature. Simon and Schuster, Inc., New York, 1984.

[2] W.C. Daywitt, A Model for Davies' Universal Superforce, Galilean Electrodynamics, vol.5, (2006) (Sept./Oct.), 83. See also www.planckvacuumDOTcom.

[3] W.C. Daywitt, The Trouble with the Equations of Modern Fundamental Physics, American Journal of Modern Physics. Special Issue: Physics without Higgs and without Supersymmetry. v. 5, (2016), no.1-1, 22.

[4] B.W. Carroll, Ostlie D.A.: An Introduction to Modern Astrophysics. Addison-Wesley, San Francisco-Toronto, (2007).

[5] W.C. Daywitt, The Planck vacuum. Progress in Physics, v. 1, 20, 2009.

[6] D.M. Gingrich, Practical Quantum Electrodynamics. CRC, The Taylor \& Francis Group, Boca Raton, London, New York, 2006.

[7] W.C. Daywitt, The Seven-Dimensional Spacetime in the Planck Vacuum Theory and the Structure of the Electron and Proton Cores, European Journal of Engineering Research and Science,Vol.5, No.4, April, 2020.

[8] R.P. Feynman, QED: the strange theory of light and matter. Princeton University Press, Princeton and Oxford, UK, 1985.

[9] R.B. Leighton, Principles of Modern Physics. McGraw-Hill Book Co., New York, Toronto, London, 1959.

[10] Julian Schwinger, On Quantum-Electrodynamics and the Magnetic Moment of the Electron. Physical Review, 1948, Vol.73, p.416. 